\title{
Geometric Bounding Techniques for Underwater Localisation Using Range-Only Sensors
}

Colin Morice, Sandor Veres (s.m.veres@soton.ac.uk)

Department of Electromechanical Engineering, University of Southampton,

Southampton, England, SO17 1BJ

Abstract This paper describes the application of geometric bounding techniques to range-only navigation of an underwater vehicle. A geometric technique is defined to obtain a position fix of an underwater vehicle using a combination of dead-reckoning navigation and acoustic measurements of range between the underwater vehicle and a GPS equipped ship. An assessment is made of the accuracy to which navigational parameters can be estimated using these methods.

Keywords: Set membership; Bounding methods; Navigation systems; Autonomous underwater vehicles.

\section{Introduction}

Typically, Autonomous Underwater Vehicles (AUVs) navigate in the underwater domain using dead-reckoning navigation systems such as bottom-lock acoustic Doppler systems and inertial navigation systems (INS). For navigation to remain accurate over long periods of time, position estimates from dead-reckoning systems must be updated periodically with measurements of absolute position, usually requiring the submersible to surface to obtain a GPS position fix. It is, however, undesirable for a submersible to routinely depart from its mission to obtain GPS fixes as surfacing is time consuming and because the accuracy of dead-reckoning navigation is much reduced while sensors are not in range of the seabed. These issues are greatly exacerbated in deep diving vehicles and so an alternative source of absolute position fix is required while navigating in the underwater domain.

As a substitute for onboard GPS, acoustic localization techniques are increasingly being used for acquisition of geo-referenced position fixes while underwater. This paper describes techniques that have been developed for analysing navigational error in the deep diving autonomous underwater vehicle Autosub 6000 [1] (Figure 1), using an acoustic localization system. Acoustic baseline navigation encompasses a number of techniques in which acoustic transponders are used to provide positioning information for underwater vehicles. The most common strategies for acoustic baseline navigation are Long Baseline (LBL) in which a number of acoustic beacons are deployed on the sea floor and localisation is achieved through triangulation like methods $[2,3$, 4], Short Baseline (SBL), in which transponders are mounted on a supporting ship [5], and Ultra-
Short Baseline (USBL), in which transponder elements are closely spaced in a single housing and are used for range-and-bearings localisation [6]. Each of these techniques requires significant effort in calibration, either in surveying the position of deployed acoustic beacons in LBL positioning or in calibration and alignment of SBL or USBL systems [7]. Recent work in range-only navigation attempts to reduce calibration issues by augmenting dead-reckoning navigation with ranging information from only a single acoustic transponder $[8,9,10,11]$.

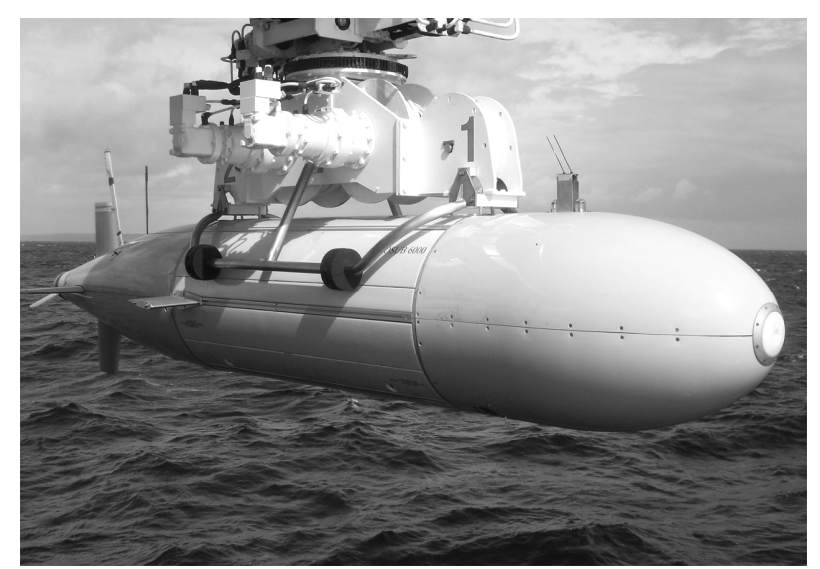

Figure 1: The Autosub 6000 autonomous underwater vehicle.

While range-only navigation has the advantage of reducing calibration requirements, careful planning of the trajectories of both the ship and submersible is required if there is to be no ambiguity in derived position fixes [10]. Without this approach, non-linear optimisation techniques such as those described in $[8,11,12]$ may converge towards local maxima.

In addition to gaining position information it is also desirable to have a measure of the error in a position derived from acoustic localisation. Recent published literature on range-only localisation $[8,9,11]$ has focused on linearised Kalman filter based range-only navigation. 
Because Kalman filter based range-only navigation requires linearisation of the range measurement equations around the filtered position estimate, the technique is sensitive to positioning errors, which may cause the filter to diverge. The largest source of these positioning errors is accumulated drift in dead-reckoning navigation while the submersible descends towards the seabed, during which acoustic Doppler based velocity sensors can only measure velocity relative to the non-stationary water. The range-only problem is therefore split into two subproblems: an initialisation step and real time filtering. As the real time filter implementation has previously been formulated as a Kalman filter, the initialisation step in $[8,11]$ is based upon optimisation yielding a point solution and Gaussian error. Such point statistic estimates may however be misleading in the presence of local maxima in the matching function if the number of measurements or data samples are small for the law of large numbers to take effect. Recent developments in filtering techniques [13], however, allow modelling of arbitrary error distributions giving rise to the possibility of alternative localisation techniques that aren't restricted to yield point solutions with Gaussian error distributions.

In this paper techniques will be outlined for obtaining error bounds on the position of an underwater vehicle by modelling error bounds on acoustic range measurements and dead reckoning navigation. Errors in all sensor measurements will be taken into account, based on the assumption that sensor errors lie within known bounds. Given conservative error bounds one can be sure that the true values of estimated parameters are contained within computed solution sets. Bounding methods for state estimation, model parameter bounding and control problems have been developed in many papers [14, 15, 16, 17]. Set membership techniques have previously been applied in the field of mobile robotics to obtain bounds on robot position with respect to known features, either to determine a robots configuration in a known environment, either as a batch operation on multiple observations of features [18, 19], or as a observers that track bounds on the changes in robot state recursively through time $[20,21,22$, $23,24,25,26]$.

Of the related work in set membership in robotics the application that is described in this paper is most closely related to that in [19], in which the position of an underwater vehicle is bounded using range measurements from multiple acoustic beacons that are mounted in floating buoys. The application in this paper differs from that described in [19] in the use of only a single acoustic beacon that is mounted on an observing ship. Based on only a single range measurement the bounded geometric set of feasible positions of the vehicle is a spherical shell centred on the ship. To further refine the submersible position set additional measurements of range between the ship and the moving submersible are required, and these range measurements will be separated in time. In order to refine the vehicle position set one must therefore also know the change in position of the submersible between consecutive range measurements. The set membership approach described in [19] will therefore be adapted so that the vehicle position set is propagated in time using dead-reckoning measurements under the assumption that the deadreckoning noise distribution is unknown but contained within known bounds. This allows the refinement of dead-reckoning position estimation using a single transceiver-transponder pair, rather than a deployed network of beacons. A technique is then developed for determining the initial position of the vehicle based on dead-reckoning measurements and measurements of range from a single acoustic beacon. This technique may be used to correct for drift in an AUV's navigation during its decent towards the seabed, during which time its navigation will be subject to increased dead-reckoning error in the form of random noise and unobservable drift due to ocean currents. Once this initial position estimate is made the AUV may navigate relative to the seabed and the observing ship is free to depart.

As a bounded error technique [16], the technique developed here yields solution sets that are guaranteed to contain the true initial position of the underwater vehicle so long as assumptions on noise bounds are satisfied. This differs from techniques based point measurements with assumptions on noise statistics as information is gained of all initial positions that are feasible given available sensory data. This is advantageous as one is not mislead about the accuracy of position estimates, as can be the case with statistical approximations of errors in point estimates.

\section{Nomenclature}

$\Delta t \quad$ Period of time between updates

$\hat{\mathbf{A}} \quad$ Estimated feasible set of $\mathbf{A}$

$\mathbf{A}^{-} \quad$ Defined as $\mathbf{A}^{-}=\{-\mathbf{a} \mid \mathbf{a} \in \mathbf{A}\}$ 
$\mathbf{A} \oplus \mathbf{B}$ Minkowski sum operator where

$\mathbf{A} \oplus \mathbf{B}=\{\mathbf{a}+\mathbf{b} \mid \mathbf{a} \in \mathbf{A}, \mathbf{b} \in \mathbf{B}\}$

d Unmeasured drift velocity in north-east-down

frame

$\mathbf{D}_{k} \quad$ Feasible set of $\mathbf{d}$

$\mathbf{E}_{d, k} \quad$ Bounds on feasible error in unmeasured drift velocity d

$\mathbf{E}_{v, k} \quad$ Bounds on feasible error in measured velocity $\mathbf{v}$

$k \quad$ Time index

$p_{n} \quad$ Time index of range measurement $n$

$\mathbf{R}_{k} \quad$ Range measurement shell, centred on position of

observing platform

S Ship position

$\mathbf{x} \quad$ AUV position

$\mathbf{X}_{0} \quad$ Initial state

$\mathbf{X}_{0}^{p} \quad$ Prior bound on $\mathbf{X}_{0}$

$\mathbf{X}_{k} \quad$ Submersible position at instant k

$\mathbf{X}_{R, k} \quad$ Position change from initial conditions

$\hat{\mathbf{X}}_{u p, k} \quad$ Range measurement updated position at instant $k$

$\mathbf{X}_{v, k} \quad$ Total measured position change since $k=0$

$\mathbf{X}_{d, k} \quad$ Total unmeasured drift since $k=0$

$\mathbf{V}_{k} \quad$ Feasible set of $\mathbf{v}$

$\mathbf{v} \quad$ Measured velocity rotated into north-east-down

frame

$\mathbf{v}^{b} \quad$ Measured velocity in body frame

$\varphi \quad$ AUV roll angle

$\theta \quad$ AUV pitch angle

$\psi \quad$ AUV yaw angle

\section{Methods}

Initially we will formulate the problem of using acoustic ranging measurements to refine a deadreckoning based estimate of the current position of an underwater vehicle. Having introduced the current position estimation problem, we then develop a technique for obtaining bounds on the initial position of an underwater vehicle. This will be done by taking range measurements while the underwater vehicle undertakes a prescribed manoeuvre.

The vehicle model used in this application is based on motion in a 3-dimensional space, in a north-east-down coordinate frame. In this reference frame the rate of change in vehicle position $\dot{\mathbf{x}}$ is defined by the rotation of a velocity vector $\mathbf{v}^{b}$, measured in a reference frame that is aligned to the submersible body, into the northeast-down coordinate frame. This is achieved by rotation around the vehicles heading, pitch and roll, which are measured by an inertial navigation system (INS).

$\dot{\mathbf{x}}=\mathbf{R}_{\text {Euler }}(\varphi, \theta, \psi) \mathbf{v}^{b}$

The dead-reckoning position estimates, determined by integration of (1), are updated by measurements of range between the submersible position $\mathbf{x}$ and the position $\mathbf{s}$ of an observing ship using a pair of acoustic transponders. Assuming spherical spreading of acoustic waves is assumed then this range $r$ is defined as

$r=\|\mathbf{x}-\mathbf{s}\|$

however, variation in sound speed as a function of depth causes acoustic waves to diffract as they propagate though the water column. Therefore, a more appropriate form of (2) is $r=g(\mathbf{x}, \mathbf{s})$, which must be solved using an acoustic ray tracing model, as described in Section 3.1.

In a set membership context the dead-reckoning based position estimate is defined as the set

$$
\hat{\mathbf{X}}_{k}=\mathbf{X}_{0} \oplus \mathbf{X}_{R, k}
$$

where $\oplus$ denotes Minkowski sum as defined in Nomenclature, $\hat{\mathbf{X}}_{k}$ is an estimated bounding set containing the submersible's current position, $\mathbf{X}_{0}$ is a set containing the submersible's initial position, $\mathbf{X}_{R, k}$ is a bounding set containing the relative change in position from the initial position and $k$ is a periodic sampling time index. Then

$\mathbf{X}_{R, k}=\mathbf{X}_{v, k} \oplus \mathbf{X}_{d, k}$

where $\mathbf{X}_{v, k}$ and $\mathbf{X}_{d, k}$ are defined as

$$
\begin{aligned}
& \mathbf{X}_{v, k}=\left(\mathbf{V}_{1} \oplus \mathbf{V}_{2} \oplus \ldots \oplus \mathbf{V}_{k-1} \oplus \mathbf{V}_{k}\right) \Delta t \\
& \mathbf{X}_{d, k}=\left(\mathbf{D}_{1} \oplus \mathbf{D}_{2} \oplus \ldots \oplus \mathbf{D}_{k-1} \oplus \mathbf{D}_{k}\right) \Delta t
\end{aligned}
$$

Here $\mathbf{V}_{k}$ is the Minkowski sum of a vector $\mathbf{v}_{k}$, the measured velocity at instant $k$ (rotated into a world coordinate frame), with a polytope $\mathbf{E}_{v, k}$, which describes the error in $\mathbf{v}_{k}: \mathbf{V}_{k}=\mathbf{v}_{k} \oplus \mathbf{E}_{v, k}$. $\mathbf{D}_{k}$ is defined by the sum of an unmeasured drift velocity $\mathbf{d}_{k}$ with a bounding error

$\mathbf{E}_{d, k}: \mathbf{D}_{k}=\mathbf{d}_{k} \oplus \mathbf{E}_{d, k}$

If the velocity sensor is tracking the sea floor and is properly calibrated then this drift component of $\mathbf{X}_{R, k}$ can be ignored. In practice $\mathbf{E}_{d, k}$ and $\mathbf{V}_{d, k}$ can defined as constant error sets, i.e. independent of $k$.

(3) therefore completely describes all possible positions of the submersible through propagation of errors in dead-reckoning navigation. This set 
can be refined by restricting the position of the submersible to be within a range bound of an observing ship. A range measurement with bounded errors can be described by a spherical set $\mathbf{R}_{k}$. The range updated location of the submersible $\hat{\mathbf{X}}_{u p, k}$ is then

$\hat{\mathbf{X}}_{u p, k}=\mathbf{R}_{k} \cap \hat{\mathbf{X}}_{k}$

and so a refined estimate of the submersible's position can be calculated from Equations (3), (4) and (7) as

$\hat{\mathbf{X}}_{u p, k}=\mathbf{R}_{k} \cap\left(\mathbf{X}_{0} \oplus \mathbf{X}_{v, k} \oplus \mathbf{X}_{d, k}\right)$

For a series of range measurements made at instants $k=p_{1} \ldots p_{N}$ the range corrected position set should be used for future dead-reckoning updates. A position update at instant $k=p_{n}$ then becomes

$\hat{\mathbf{Y}}_{u p, p_{n}}=\mathbf{R}{ }_{p_{n}} \cap\left(\hat{\boldsymbol{X}}_{u p, p_{n-1}} \oplus \mathbf{X}_{v, p_{n}} \oplus \mathbf{X}_{d, p_{n}}\right)$

Where $\hat{\mathbf{X}}_{u p, p_{n-1}}$ is the range updated position set at $k=p_{n-1}$, and $\mathbf{X}_{v, p_{n}}$ and $\mathbf{X}_{d, p_{n}}$ bound the accumulated observed dead-reckoning position changes and unmeasured drifts from the instant of the previous range measurement $k=p_{n-1}$ to the instant of the current position estimate $k=p_{n}$ such that

$$
\begin{array}{r}
\mathbf{X}_{v, p_{n}}=\left(\mathbf{V}_{p_{n-1}} \oplus \mathbf{V}_{p_{n-1}+1} \oplus \ldots \oplus \mathbf{V}_{p_{n}-1} \oplus \mathbf{V}_{p_{n}}\right) \Delta t \\
\mathbf{X}_{d, p_{n}}=\left(\mathbf{D}_{p_{n-1}} \oplus \mathbf{D}_{p_{n-1}+1} \oplus \ldots \oplus \mathbf{D}_{p_{n}-1} \oplus \mathbf{D}_{p_{n}}\right) \Delta t
\end{array}
$$

It should be noted that for highly vertical geometries (submersible depth much greater than the horizontal ship-submersible distance) the range measurement set can be approximated as a spherical shell centred on the observing ship corrected for average sound speed using standard tables such as Carter's tables [27]. If this is not the case then the range measurement must be constructed using a sound propagation model using a sound speed profile obtained in situ. The influence of GPS errors on the range measurement set can be included in calculations by Minkowski addition of a set describing uncertainty of ship position to the range set.

\subsection{The use of range measurements to bound the initial position of a vessel}

To determine a bounding set for the submersible's initial position we wish to find a set $\hat{\mathbf{X}}_{0}$ encompassing all possible initial positions for which a solution to (8) exists. Formally this is described by (12)

$\hat{\mathbf{X}}_{0, k}=\left\{\mathbf{x}_{0} \mid \mathbf{R}_{k} \cap\left(\mathbf{x}_{0} \oplus \mathbf{X}_{v, k} \oplus \mathbf{X}_{d, k}\right) \neq \varnothing\right\}$

Now we note that Minkowski addition can be described in an alternative form as

$$
\mathbf{A} \oplus \mathbf{B}=\{z \mid \mathbf{A} \cap(z-\mathbf{B}) \neq \varnothing\}
$$

Bearing (13) in mind we see that (12) can be rewritten as

$\hat{\mathbf{X}}_{0, k}=\left\{\mathbf{x}_{0} \mid \mathbf{R}_{k} \cap\left(\mathbf{x}_{0}-\mathbf{X}_{R, k}^{-}\right) \neq \varnothing\right\}$

where

$\mathbf{X}_{R, k}^{-}=\left\{-\mathbf{x} \mid \mathbf{x} \in\left(\mathbf{X}_{v, k} \oplus \mathbf{X}_{d, k}\right)\right\}$

(12) can therefore be expressed as

$$
\hat{\mathbf{X}}_{0, k}=\mathbf{R}_{k} \oplus \mathbf{X}_{R, k}^{-}
$$

The best bound $\hat{\mathbf{X}}_{0, k}$ for the initial position based on the range measurement at instant $k$ is given by (14). When range measurements are available at a number of different instants $k$ the true initial condition is bounded by $\hat{\mathbf{X}}_{0, k}$ for all $k$. The true initial position can therefore be bounded by

$$
\hat{\mathbf{X}}_{0}=\bigcap_{k=1}^{K}\left(\mathbf{R}_{k} \oplus \mathbf{X}_{R, k}^{-}\right)
$$

Further, if a prior bound $\mathbf{X}_{0}^{p}$ on $\mathbf{X}_{0}$ is known then

$$
\hat{\mathbf{X}}_{0}=\mathbf{X}_{0}^{p} \bigcap\left(\bigcap_{k=1}^{K}\left(\mathbf{R}_{k} \oplus \mathbf{X}_{R, k}^{-}\right)\right)
$$

(18) allows estimation of the initial position of a submersible based on ship-submersible range measurements and dead-reckoning velocity information. Here $\mathbf{X}_{0}^{p}$ may describe an initial position derived from either propagation of deadreckoning measurements taking into account 1) drift, 2) bounds on vehicle depth obtained from a pressure sensor, or 3) the full region bounded by the sea floor and surface.

\section{Simulation of Geometric Techniques using Artificial Data}

\subsection{Simulation setup for estimation of initial position of a vessel}

The techniques described in Section 2 have been applied to simulated navigational data using the Geometric Bounding Toolbox (GBT) [28]. Modelled error bounds in measurements and real world parameters take values that are consistent with those that would be encountered in real world 
operation (see Table 1). Values quoted are consistent with those stated in [29]. As this paper represents part of a project to develop navigational tools for the deep diving underwater vehicle, Autosub 6000, error parameters have been chosen that most accurately model errors in Autosub's sensors. Autosub 6000, being equipped with an IXSEA PHINS inertial navigation system, can be expected to experience attitude error of up to $0.02^{\circ}$. This corresponds to a positioning error that is two orders of magnitude smaller than can be expected of the Acoustic Doppler Current Profiler (ADCP) used by Autosub 6000 to measure velocity, which is a Teledyne RD Instruments Workhorse Navigator. Because of this, errors in INS attitude measurements have been neglected. As the intended application of the initial position estimation technique is the correction of drift in dead-reckoning navigation by re-initialisation of vehicle position once it has descended to within sensor range of the ocean floor, the unobservable component of drift velocity defined in (6), assumed to be due to ocean currents, can be ignored as velocity is measured by the ADCP with respect to the static seabed. Therefore, so long as the vehicle is within sensor range of the seabed and a set that bounds the unmeasured component of velocity the set bounding this drift velocity is $\mathbf{D}=\mathbf{0}$. The vehicle velocity is then described by $\mathbf{V}$, which is modelled as an orthotope that bounds the set of feasible velocities measured by the $\mathrm{ADCP}$, rotated into the north-east-down coordinate frame. The range error used is that quoted by LinkQuest, the manufacturers of the TrackLink 10000 acoustic transponder system that is installed on Autosub 6000. The bounds in GPS position fixes are taken at $99.7 \%$ confidence intervals of fix accuracy seen in GPS data. Error bounds on depth measurements are chosen to bound errors in measurements obtained using an onboard pressure sensor.

Table 1: Errors used in simulations

\begin{tabular}{|c|c|}
\hline \multicolumn{2}{|c|}{ Simulation Errors } \\
\hline $\begin{array}{c}\text { ADCP velocity } \\
\text { error }\end{array}$ & $\pm 0.3 \%$ distance travelled \\
\hline INS error & $<0.02 \mathrm{deg}$ (negligible) \\
\hline Range error & $\pm 0.4 \mathrm{~m}$ \\
\hline Ship GPS error & $\pm 1.23 \mathrm{~m}$ \\
\hline Depth error & $\pm 1 \mathrm{~m}$ \\
\hline
\end{tabular}

For proper application of the system the effect of a non-constant sound speed on the propagation of acoustic waves should be taken into account. The ray tracing model is used to model the propagation of the acoustic waves away from the acoustic source by successive application of Snell's Law as rays travel through each layer in an ocean that is assumed to be vertically stratified with respect to sound speed. Within each layer in the vertically stratified ocean a linear variation in sound speed is assumed. Under this assumption acoustic rays will travel along arcs of circles through each sound speed layer in the model. By tracing the paths of rays emitted from the acoustic source at a number of angles two isotemporal curves are formed describing the inner and outer bounds on the range shell. It should be noted that these isotemporal curves would generally not be spherical except in the case that the sound speed is constant across all depths.

In this paper a ray-tracing model has been used to simulate the effects of varying sound speed on acoustic range measurements for sound speed profiles that can be expected in North Atlantic and in Antarctic regions. These sound speed profiles represent the extremes of sound speed variation that Autosub 6000 is likely to encounter in operation. The sound speed profiles used are shown in Figure 2. The sound speed profile will affect the size of the observed solution set by affecting the angle at which acoustic wave fronts arrive at the submersible and will also have a small affect on the bounds of the range shell.

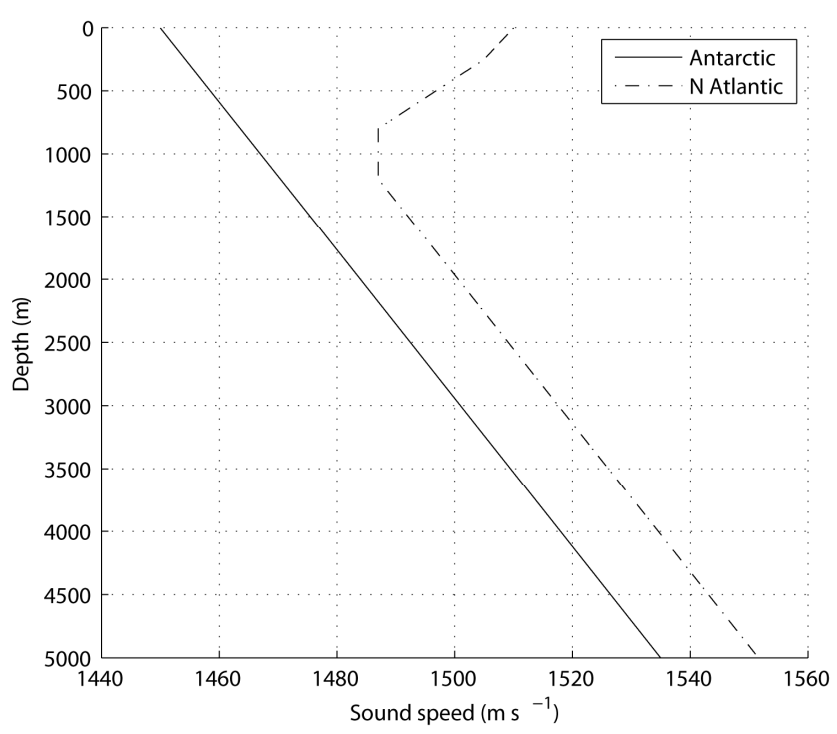

Figure 2: Sound speed profiles used in simulations.

The approach adopted in this paper for computation of the operations described in Section 2.1 is based on conducting operations on convex polytopes using the GBT [28,31,32]. This requires that the range shell set is approximated by 
a set of convex polytopes that together contain the range shell. Each of these polytopes are formed by hyperplanes describing upper and lower bounds on the depth measurement, inner and outer bounds on the range measurement isotemporal curves under the assumption of plane wave propagation within the depth bounds and a pair of hyperplanes that bound an arc of rotation around the vertical depth axis. This approximation is demonstrated in Figure 3, where the range shell is approximated by a set of 8 polytopes, each of which spanning an arc of 45 degrees around the depth axis. The approximation of the range shell used in simulations is split into 90 segments, each spanning an arc of 4 degrees around the depth axis. In this representation of the range measurement the Minkowski addition $\hat{\mathbf{X}}_{0, k}=\mathbf{R}_{k} \oplus \mathbf{X}_{R, k}^{-}$is computed by adding $\mathbf{X}_{R, k}^{-}$to each convex polytope in $\mathbf{R}_{k}$, while intersections evaluated in (17) and (18) are computed as the set intersections of each polytope in $\mathbf{R}_{k} \oplus \mathbf{X}_{R, k}^{-}$with each polytope in $\hat{\mathbf{X}}_{0, k-1}$. There is therefore a progressive increase in the complexity of the solution polytope $\hat{\mathbf{X}}_{0}$ as additional measurements are used to update the solution set. This increase in computational complexity can be mitigated by applying a simplification step to reduce polytope complexity. The solution set can be approximated using bounding convex polytopes, for example using outer bounding parallelotopes [22] or minimum volume ellipsoids [23], after each measurement is used to update $\mathbf{X}_{0}$. Simplifications of this form are essential in realtime state estimation, as discussed in [21]. In the application described in this paper no such approximations have been applied. This limitation restricts the applicability to estimation of the initial position set from only a small number of range measurements. However, by approximating the position set with an outer bounding polytope after each range measurement, the described technique can be used in recursive real-time estimation.

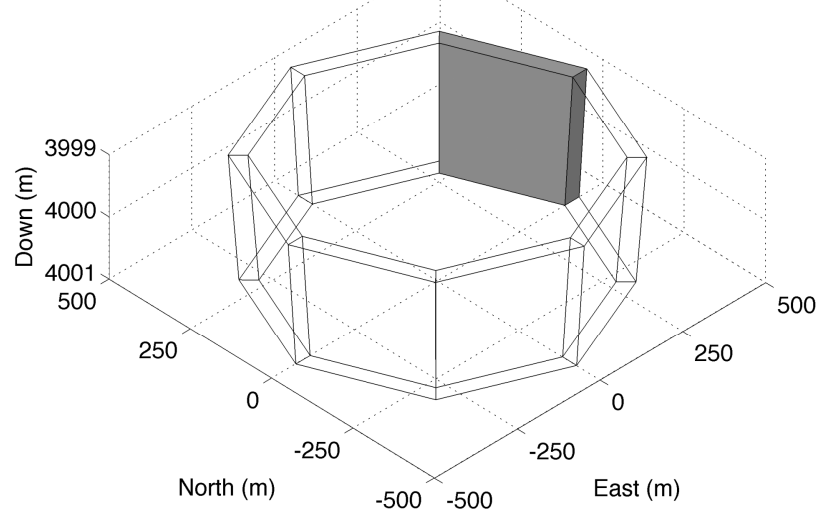

Figure 3: An example of the polygonal approximation to the range set, shown as a wire frame, limited by upper and lower bounds on vehicle depth. The approximation is constructed as a set of convex polytopes, one of which shaded in grey, that outer bound the range measurement set. The example approximation shown here is constructed from 8 convex polytopes. Approximations used in simulations are constructed in a similar manner from 90 convex polytopes.

\subsection{Simulation results}

Simulations have been carried out to estimate the initial position based on (18). The strategy employed in simulations is to obtain acoustic range measurements while the submersible navigates along the perimeter of a box that is centred on the supporting ship. An example of one such navigation box is shown in Figure 4. If a trajectory of constant heading were used, instead of the perimeter of the box, the solution set would consist of a pair of unconnected sets lying either side of the submersible's trajectory. While the bounding technique described here will allow the estimation of a solution comprised of a pair disparate subsets, such a solution is undesirable and so the trajectory in which the vehicle navigates around the perimeter of a box has to been chosen as it allows a single solution set to be determined. An example solution for bounding the feasible set of initial positions based on navigating around this "box trajectory" is shown in Figure 5. Each ring depicted in the left plot of Figure 5 is a set of initial positions that are feasible given a single range measurement and bounds on the vehicle depth. The true initial position must be consistent with all sensor measurements and so must lie within the intersection of each of these rings. This intersection is shown in the right plot of Figure 5. 


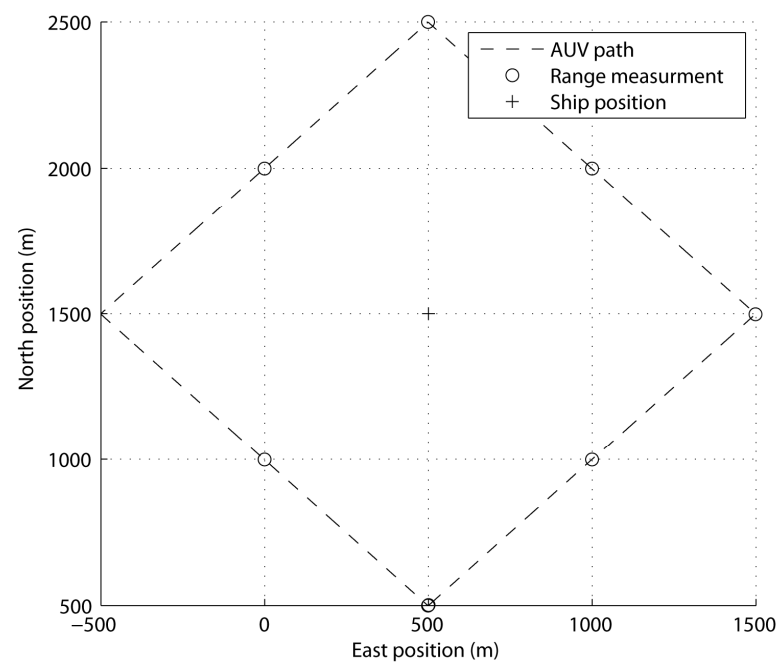

Figure 4: An example trajectory used for localization. The submersible begins at position $(500,500)$ and navigates a box of side length $1000 \mathrm{~m}$ around the ship, located at the origin, while ship-to-submersible range is measured acoustically.
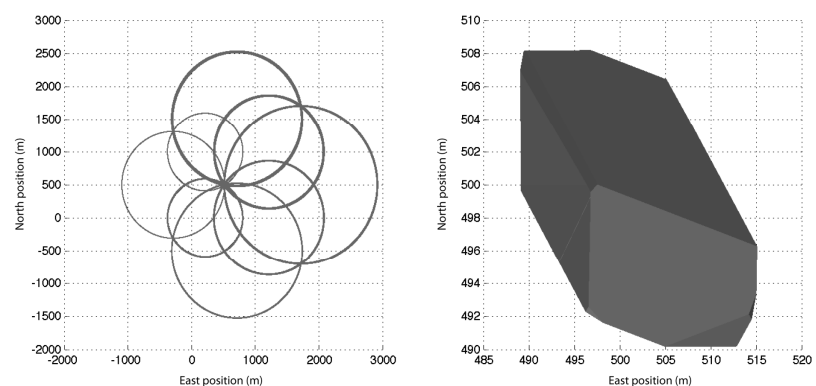

Figure 5: Initial position estimation for a vehicle navigating around along the path shown in Figure 4. Left Panel: each shaded ring contains all feasible positions of the submersible at $k=0$ given a single range measurement and all deadreckoning data up to the instant of the range measurement. Right Panel: the intersection of the rings shown in the left panel corresponding to the feasible set of initial positions

$\mathbf{X}_{0}$ given all available data, as computed from (18).

It is desirable to have a measure of the accuracy that can be expected for a given box size at a given depth. To this end, navigational boxes similar to that shown in Figure 4 have been simulated for boxes of side length ranging from $250 \mathrm{~m}$ to $1500 \mathrm{~m}$, and at depths ranging from 500 $\mathrm{m}$ to $4000 \mathrm{~m}$. Error parameters used in simulations are those shown in Table 1. For all simulations the resulting initial position sets contain the true initial positions. The sizes of the initial position set calculated in each simulation are shown in Figure 6. These sizes are the lengths of the maximum dimension across the initial position set calculated in each simulation. It can be seen that the solution size is relatively insensitive to sound speed profile so long as sound speed information is accurate, with a difference between solution size observed in the North Atlantic and Antarctic waters of less than $0.5 \mathrm{~m}$ in all simulations. Figure 6 shows that the size of the solution sets is dependent on the size of the box around which the submersible navigates. If the submersible navigates around the perimeter of a box of short side length then accumulated deadreckoning error is small but the acoustic wave fronts of the range measurement impinge on the submersible at a shallow angle to the horizontal plane so that the range measurement provides little information of the horizontal position of the submersible. The converse becomes true with increasing navigation box size as range measurements intersect with the vehicle position set at a steeper angle to the horizontal but bounds on dead-reckoning error increase due to the larger distance travelled. Figure 6 indicates that there is an optimal size for the box at which these two factors balance to give a minimum size feasible set of initial positions.

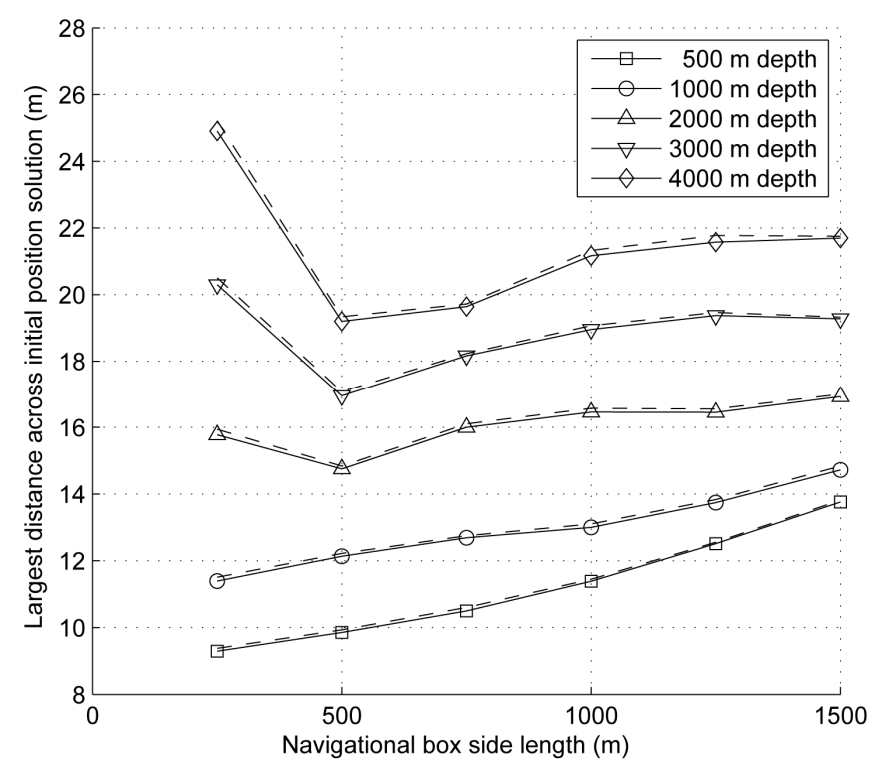

Figure 6: Size of initial position solution $\mathbf{X}_{0}$ corresponding for boxes with sides of length $250 \mathrm{~m}$ to $1500 \mathrm{~m}$ calculated in $250 \mathrm{~m}$ steps at depths of $500 \mathrm{~m}$ to $4000 \mathrm{~m}$. Results for Antarctic sound speed conditions are shown as solid lines while North Atlantic conditions are shown as dashed lines.

It should be noted that at present there are some limitations introduced by the computational techniques used to produce the results shown in Section 3. As calculations are currently based on vertex and polytope facet calculations, the input and output sets can only be described as straight edged, polygonal sets. There is therefore some approximation of the range measurement sets, which are inherently curved. The computational techniques currently used are also limited to application in short term estimation of vehicle position, as solution sets become increasingly complex as additional range measurement 
information is incorporated. These limitations can, however, be mitigated by employing a polytope simplification strategy in which the solution set is approximated by an outer bounding polytope of simpler form, as discussed in Section 3.1. A further limitation of the system described here is the sensitivity of the geometric techniques used to outliers in measurements. Techniques for dealing with outliers in geometric computation are documented in [30] and there has been promising recent work [25] in bounded parameter estimation with a fixed number of outliers. It is clear that the techniques described in this paper should be extended to allow for potential outliers to allow robust application of the system.

\section{Conclusions}

The bounding techniques described in this paper have a number of advantages over likelihood based range-only submersible positioning described in the literature [8, 11]. Firstly, the computed solution sets may be disparate and so can show multiple discrete solutions. Secondly, no assumptions are made about sensor noise statistics other than that sensor noise lies within known tolerances. Thirdly, the full range of possible solutions that are consistent with measurement errors are calculated, rather than the single most likely solution with a lower bound on error variance $[8,11]$. This set of solutions can, however, be expected to appear to be more conservative than statistical likelihood methods that exploit assumed statistical properties of errors not certain to be valid in reality.

While the techniques described here have been applied to problems in underwater navigation they can equally well be applied to localisation in terrestrial navigation with respect to known landmarks. In this setting, the bounds on the initial location of a robot can be computed based on computation of the equations described in Section 2. We believe that the techniques described here have potential as useful tools both in their own right and for validation of navigational parameters estimated by other methods. It is hoped that the results shown in Figure 6 will serve as useful guidelines to the accuracy that can be achieved in singletransponder pair range-only navigation of vehicles operating at full ocean depth.

As discussed in Section 3, the system described in this paper is currently sensitive to outliers in range measurements. The development of parameter bounding techniques, that are robust to outliers, is an ongoing research area and it is believed that further work should focus on this issue. The technique described in this paper should also be extended to allow estimation of additional navigational parameters such as heading errors and biases related to calibration of the deadreckoning system. Finally, developments of complexity reduction techniques by approximate polytopes have the potential to enhance the speed of real-time computations. Approximations can be used to obtain the bounding sets of position estimates, similarly to the approximations used in [31].

\section{Acknowledgements}

This work has been jointly funded by the Underwater Systems Laboratory, National Oceanography Centre, Southampton, the School of Engineering Sciences, University of Southampton, Southampton and the Engineering and Physical Sciences Research Council (EPSRC) Grant No. EP/E02677X/1.

\section{References}

[1] McPhail, S.D. RRS Discovery Cruise 323, 19 September-3 October 2007, First Deepwater Trials of the Autosub6000 AUV, Technical report, National Oceanography Centre, Southampton, 2008.

[2] Hunt, M.M., Marquet, W.M., Moller, D.A., Peal, K.R., Smith, W.K., and Spindel, R.C. An acoustic navigation system. Technical Report, Woods Hole Oceanographic Institution, 1974. [3] Milne, P.H. Underwater acoustic positioning systems. Gulf Publishing Co., Houston, TX, 1983.

[4] Whitcomb, L.L., Yoerger, D.R., Singh, H., and Howland, J. Combined Doppler/LBL based navigation of underwater vehicles. Proc. Int. Symp. on Unmanned Untethered Submersible Technology, 1999.

[5] Bingham, B., Mindell, D., Wilcox, T., and Bowen, A. Integrating precision relative positioning into Jason/Medea ROV operations. Marine Technology Society Journal, 2006, 40(1), 8796.

[6] Morgado, M.,Oliveira, P.,Silvestre, C., and Vasconcelos, J.F. USBL/INS Tightly-Coupled Integration Technique for Underwater Vehicles. Proceedings Of The 9th International Conference on Information Fusion, 2006.

[7] Vickery, K. Acoustic positioning systems. A practical overview of current systems. Proceedings Of The 1998 Workshop on Autonomous Underwater Vehicles, AUV'98, 1998, 5-17.

[8] Eustice, R.M., Whitcomb, L.L., Singh, H., and Grund, M. Experimental results in synchronous-clock one-way-travel-time acoustic navigation for autonomous underwater vehicles.

Proceedings of the IEEE International Conference on Robotics and Automation, Rome, Italy, 2007, 4257-4264.

[9] Larsen, M.B. Synthetic long baseline navigation of underwater vehicles. OCEANS 2000 MTS/IEEE Conference and Exhibition, 2000, 3.

[10] Scherbatyuk, A.P. The AUV positioning using ranges from one transponder LBL. OCEANS'95. MTS/IEEE. 'Challenges of Our Changing Global Environment'. Conference Proceedings, 1995, 3. [11] Baccou, P., and Jouvencel, B. Simulation results, postprocessing experimentations and comparisons results for navigation, homing and multiple vehicles operations with a new 
positioning method using on transponder. IEEE/RSJ International Conference on Intelligent Robots and Systems, 2003.(IROS

2003,.2003, 1.

[12] Newman, P., and Leonard, J. Pure range-only sub-sea

SLAM. IEEE International Conference on Robotics and

Automation, 2003. Proceedings. ICRA'03, 2003, 2.

[13] Arulampalam, M.S., Maskell, S., Gordon, N., Clapp, T.,

Sci, D., Organ, T., and Adelaide, S.A. A tutorial on particle

filters for online nonlinear/non-GaussianBayesian tracking. IEEE

Transactions on signal processing, 2002, 50(2), 174-188.

[14] Walter, E. Special issue on parameter identification with error bounds. Mathematics and Computers in Simulation, 1990, 32(5-6), 447-607.

[15] Kurzhanski A.B., and Veliov V.M. Modeling Techniques for Uncertain Systems, Progress in Systems \& Control Theory.

Birkhäuser/IIASA, Boston, 1994.

[16] Milanese, M., Norton, J., Piet-Lahanier, H., and Walter, E. Bounding Approaches to System Identification, Plenum Press,

New York, NY, USA, 1996.

[17] Jaulin, L., Kieffer, M., Didrit, O., and Walter, E. Applied

Interval Analysis, Springer London, 2001.

[18] Jaulin, L., Walter, E., Leveque, O., and Meizel, D. Set inversion for chi-algorithms, with application to guaranteed robot localization. Mathematics and Computers in Simulation, 2000,

52(3), 197-210.

[19] Caiti, A., Garulli, A., Livide, F., and Prattichizzo, D.

Localization of autonomous underwater vehicles by floating acoustic buoys: a set-membership approach. IEEE Journal of Oceanic Engineering, 2005, 30(1), 140-152.

[20] Di Marco, M. Set-membership estimation techniques for mobile robotics applications, $\mathrm{PhD}$ Thesis, Università degli Studi di Bologna, Firenze, Padova, Siena, 2001.

[21] Di Marco, M., Garulli, A., Giannitrapani, A. and Vicino, A. A set theoretic approach to dynamic robot localization and mapping. Autonomous Robots, 16(1), 23-47, 2004.

[22] Garulli, A. and Vicino, A. Set Membership localization of mobile robots via angle measurements, IEEE Transactions on Robotics and Automation, 17(4), 450-463, 2001.

[23] Hanebeck, U.D., and Schmidt, G. Localization of fast mobile robots based on an advanced angle-measurement technique, Control Engineering Practice, 4(8), 1109-1118, 1996.

[24] Jaulin, L., Kieffer, M., Walter, E. and Meizel, D. Guaranteed robust nonlinear estimation with application to robot localization, IEEE Transactions on Systems, Man, and Cybernetics, Part C, 32(4), 374-381, 2002.

[25] Jaulin, L. Robust set membership state estimation; Application to underwater robotics, Automatica, 45(1), 202-206, 2009.

[26] Jaulin, L. A nonlinear set membership approach for the localization and map building of underwater robots, IEEE Transactions on Robotics, 25(1), 88-98, 2009.

[27] Carter, D.J.T. Echo-sounding correction tables.

Hydrographic Department, Ministry of Defence, Taunton, UK, NP, 1980, 139-150.

[28] Veres, S.M. Geometric Bounding Toolbox (GBT) for Matlab. Official website: http://www. sysbrain.com/gbt, 2003.

[29] Kinsey, J.C., Eustice, R.M., and Whitcomb, L.L. A survey of underwater vehicle navigation: recent advances and new challenges. Proceedings of the 7th Conference on Maneuvering and Control of Marine Craft (MCMC'2006), IFAC, Lisbon, 2006.

[30] Bai, E.W., Cho, H., Tempo, R., and Ye, Y. Optimization with few violated constraints for linear bounded error parameter estimation. IEEE Transactions on Automatic Control, 2002 47(7), 1067-1077.

[31] Veres, S. M. Error control in polytope computation. Journal of Optimization Theory and Applications, 2002, 113(2), 325-355. [32] Veres, S. M., Gabriel, S.B., Rogers, E. and Mayne, D. Q. Analysis of formation flying control of a pair of nanosatellites. AIAA Journal of Guidance, Control and Dynamics, 2002, 25(5), 971-974. 